\title{
Deficits of heterozygotes in relation to selective interactions between loci in the mussels Brachidontes rostratus and Xenostrobus pulex
}

\author{
D. J. Colgan
}

\author{
Department of Evolutionary Biology, \\ Research School of Biological Sciences, \\ G.P.O. Box 475, Canberra City, Australia 2601.
}

Each marine mussel which has been studied has a polymorphism for leucine aminopeptidase ( $L A P)$ loci. In each case, there is a deficit for all, or nearly all, classes of heterozygotes. The consistency of the results suggests that the loci constitute non-transient polymorphisms with heterozygous disadvantage. No satisfactory explanation of this situation has been available. Heterozygous deficits are also often found at other loci in mussels, particularly those encoding aminopeptidase $(A P)$ enzymes. The present work was prompted by the theoretical investigations of Hastings (1982) which revealed the possibility that selective interactions with other loci might enable the maintenance of polymorphisms with marginal underdominance. Non-random associations between genotypes were sought in 10 loci (in four groups of enzymes with related functions) in Brachidontes rostratus and five loci (in two groups) in Xenostrobus pulex. Five of the 30 pairs of loci displayed significant genotypic associations. However, none of these comprised functionally-related enzymes. It remains possible that epistatic interactions may, in special circumstances, be involved in the maintenance of variation. But the process seems unlikely as a general explanation of genotypic frequencies of the $L A P$ and $A P$ polymorphisms of mussels.

\section{INTRODUCTION}

Enzyme electrophoresis has revealed a plethora of genetic polymorphism in an extreme diversity of organisms. Yet there are few examples of loci where genotypes have plausibly been associated with differential fitness. The leucine aminopeptidase $(L A P)$ polymorphisms of marine bivalves are prominent among these examples because of the consistent deviations from Hardy-Weinberg expectations which they exhibit. In each species studied, loci encoding these enzymes show significant deficits of heterozygotes. Among the 30 or so species which have been examined (Foltz, 1986) are Mytilus edulis (Koehn, Milkman and Mitton, 1976; Skibinski, Beardmore and Cross, 1983), $M$. californianus (Levinton and Suchanek, 1978), $M$. galloprovincialis (Skibinski, Beardmore and Cross, 1983), Brachidontes rostratus (Colgan, 1981), Crenomytilus grayanus (Kartavtsev, 1978) and Guekensia demissa (Garthwaite, 1986) from the mytilid family. Heterozygous deficits are often found for other loci, particularly for those encoding aminopeptidase $(A P)$ enzymes (Koehn, Milkman and Mitton, 1976), although the patterns for these are not as uniform as that of $L A P$. The consistency of the $L A P$ results challenges the theory of population genetics to show how polymorphisms with disadvantageous heterozygotes could be maintained. Many possible explanations of these observations have been recognized. But none has been convincingly demonstrated (Colgan, 1981; Zouros and Foltz, 1984). The uniformity of genotypic frequencies between sexes disallows the general involvement of sex-linkage (Koehn, Milkman and Mitton, 1976; Garthwaite, 1986). Breeding studies tend to discount the possibility of operation of some process whereby alleles are not expressed in a co-dominant manner in heterozygotes (Beaumont, Beveridge and Budd, 1983; Adamkewicz, Taub and Wall, 1984; Mallet et al., 1985) although data on $F_{2}$ and backcross generations are required before this point is proven. Colgan (1984) has shown that selection at loci in linkage disequilibrium is unlikely to cause significant segregation ratio distortions in pedigree analysis. So the breeding studies referred to above argue against the possibility that the deviations from Hardy-Weinberg expectations at mussel loci are due to selection at non-studied loci in linkage disequilibrium. 
There has been a report of the possible identification of null alleles at the LAP-2 locus of the oyster, Crassostrea virginica (Foltz, 1986). But the genotypic frequencies observed at $L A P$ loci often do not fit the expectations derived from the assumption that null alleles are the sole cause of the heterozygous deficits (Colgan, 1981). This is also the case, for at least some species, for the expectations amended for the undoubted operation of the Wahlund effect (Koehn, Milkman and Mitton, 1976; Gartner-Kepkay et al., 1980; Colgan, 1981; Skibinski, Beardmore and Cross, 1983). This is because there is too little variance in genotypic frequencies between the sub-populations of the species.

Colgan (1981) considered three further explanations of the apparent contradiction between heterozygous deficits and widespread polymorphism. These are frequency-dependent selection in favour of rare genotypes and temporal or geographic differences in the superiority (or inferiority) of the various homozygotes. No data are available to test directly the first of these possibilities. Such data would be of great value in view of the possibility that frequency dependent selection can result in stable polymorphism even if the heterozygote is less fit than either homozygote (Lewontin, 1958). There is evidence that spatial differences in the relative fitness of homozygotes are important in the maintenance of genetic variation in the LAP loci of M. edulis (Koehn, Milkman and Mitton, 1976; Theisen, 1978; Hilbish, 1985). But such differences did not give predictions which fitted the data from B. rostratus (Colgan, 1981). Moreover, Beaumont, Beveridge and Budd (1983) and Mallet et al. (1985) have demonstrated that heterozygous deficits occur in the progeny of single-pair matings conducted in the laboratory. If this is due to selection discriminating between genotypes rather than to the repression of an allele and if it is generally the case in marine populations then, although the relative fitness of homozygotes may be variable, the polymorphisms should be transient.

It would be premature to dismiss all of the above possibilities as sole or joint cause of the maintenance of polymorphisms with apparent heterozygote disadvantage. There must be, however, a strong suspicion that the true basis of this maintenance remains to be discovered. A promising line of research has recently been opened by the analytical demonstration that multi-locus systems with constant fitness and marginal heterozygote deficits can be stably maintained given the appropriate conditions of epistatic selection
(Hastings, 1982). The examples provided by Hastings are considered by him to be biologically unusual because they require that the fittest homozygote should be in a very low frequency. Nevertheless, he has investigated only a small proportion of the possible combinations of two-locus genotypic frequencies. Other frequency/fitness combinations which lead to the maintenance of polymorphisms with apparent marginal heterozygous disadvantage may be discovered. Some of these may be more biologically realistic.

The first step in the investigation of this line of research is to search for non-random associations between genotypes at different loci. Two studies of this point have been made within single species of marine mussels. Mitton and Koehn (1973) reported a significant association between genotypes at a $L A P$ locus and an aminopeptidase $(A P)$ locus in $M$. edulis. Ahmad and Hedrick (1985) studied four enzymes, including $L A P$ and $A P$, in $M$. edulis. An association of $L A P$ with the functionally unrelated enzyme phosphoglucomutase was found but there was no evidence of a significant interaction between the $L A P$ and $A P$ loci. There are two main reasons for continuing such work. The first is to reconcile the differences in the results of the available studies. Evidence from other species may clarify which of the two results is more general. Secondly, a wider range of enzymes should be examined for genotypic interactions as, in the context of Hasting's model, it can be argued that the relevant interactive locus has not been identified. This is particularly the case for the aminopeptidases. It seems that only one type of (dipeptide) substrate has been used in the detection, after electrophoresis, of such enzymes in M. edulis (Mitton and Koehn, 1973; Ahmad and Hedrick, 1985). In vertebrates, a number of quite distinct enzymes are known to have peptidolytic activity against characteristic suites of di-, tri- and oligo-peptides (Harris and Hopkinson, 1977). This may also be true of some mussel species.

The present study was undertaken to test whether there is any non-random association of the genotypes of different loci in two species of mussel, B. rostratus and Xenostrobus pulex. Theoretically, selective interactions could occur between any two loci. Functional relationship between loci was considered the best criterion for choosing which loci to investigate. The primary aim of the study was to search for associations between such loci. However, the degree of association of enzymes which were not functionally related was also determined because this may provide information about 
linkage between loci and about the level of frequency distortions caused by the Wahlund effect.

\section{MATERIALS AND METHODS}

Mussels were collected by Dr G. Brand from single clumps less than two square metres in size. Brachidontes rostratus was collected from the population at Barwon Heads studied by Colgan (1980) and Xenostrobus pulex was collected from a population at Point Lonsdale near the site of the $B$. rostratus population described by Colgan (1980). On arrival at the laboratory, mussels were frozen at $-90^{\circ} \mathrm{C}$ until required for electrophoresis.

Previous electrophoretic studies of B. rostratus (Colgan, 1981) had used acrylamide as the gel medium. In this study, however, cellulose acetate ("Cellogel", Chemetron, Milan) was utilised. This was primarily because of its superior resolution: seven allozymes being discernible for $L A P$ whereas only four had been separated by any combination of buffer and gel characteristics using the previous methods.

Details of grinding buffers and electrophoretic buffers and procedures are given in Colgan (1986). For electrophoresis, all of the soft tissues of the mussels were ground in hand-held glass homogenisers. One volume of buffer (approximately) was used for one volume of tissue. The homogenate was centrifuged in an Eppendorff centrifuge for 4 minutes to remove cellular debris. The supernatant was used for electrophoresis. Aliquots were immediately placed at $-90^{\circ} \mathrm{C}$ for later re-testing in comparison gels.

The mussels used in the experiments ranged between 8 and $20 \mathrm{~mm}$ for $B$. rostratus and 6 and $12 \mathrm{~mm}$ for $X$. pulex. Mussels larger than these sizes were not found in the samples whilst smaller individuals were discarded. Analyses of the size distributions of B. rostratus (Colgan, 1980) suggest that $B$. rostratus in the range used here would probably derive from one or two annual cohorts. This may also be the case for the $X$. pulex sample which had an unimodal size distribution in the range of tested mussels. However, no other data are available to support this.

A number of enzymes were scored in a preliminary survey of five $B$. rostratus individuals which was performed to test the suitability of cellulose acetate. Ten were chosen for further study in this species. These were all well localised on the gel. All but two were polymorphic in the survey. The two invariant enzymes were included in the larger scale study because of their functional relationship with others which were to be scored. The enzymes which were selected for B. rostratus are given, with their abbreviations, in the following list. Phosphoglucomutase (PGM), mannosephosphate isomerase (MPI) and glucosephosphate isomerase $(G P I)$ are functionally related in that the end product of each of the first two is a substrate for the third. Glucose-6-phosphate dehydrogenase $(G-6-P D H)$ and 6-phosphogluconate dehydrogenase (6-PGDH), which comprise the first pair of enzymes on the phosphogluconate oxidation pathway, are related to the previous group of enzymes because glucose-6-phosphate, the endproduct of $P G M$, is the substrate for G-6-PDH. Glyceraldehyde-3-phosphate dehydrogenase ( $G A$ 3-PDH) and $\alpha$-glycerophosphate dehydrogenase $(G P D)$ respectively reduce and oxidize equivalent amounts of the co-factor NAD midway through the process of glycolysis. All of the above enzymes were stained according to the methods detailed in Colgan (1986). The staining methods for the remaining group of enzymes, the peptidases, are outlined below. Leucine aminopeptidase (LAP) was stained by blotting the gel for five minutes in $1 \mathrm{ml}$ of $0.2 \mathrm{M}$ tris- $\mathrm{HCl}, p \mathrm{H} 5.5$ containing $1 \mathrm{mg}$ of 1-leucyl-4-methoxy- $\beta$-napthylamide. The gel was then dipped in $1 \mathrm{ml}$ of the same buffer containing $5 \mathrm{mg}$ of Fast Black K salt. Aminopeptidases were stained according to Harris and Hopkinson (1977). The peptides which were tested in the preliminary survey were leucine-proline, leucine-alanine, leucine-glycine-glycine and valine-leucine. No enzyme was detectable with leucine-proline. Three loci were visible on gels stained with leucinealanine or leucine-glycine-glycine. The most anodal of these was $L A P$. The second-most anodal was more active against the latter substrate so it is referred to below as $L G G$. The third enzyme was the most active of all the peptidases against both leucine-alanine and leucine-glycine-glycine. It was relatively more active, as judged by visual inspection, against the former and was clearly variable. This variation was not, however, consistently interpretable so this locus was ignored in data analysis. Apparent heterozygotes for $L A P$ and $L G G$, in common with previously-reported variation for aminopeptidases in other mussels, possess only two bands. This suggests that their functional form is monomeric, although it has been stated (Young, Koehn and Arnheim, 1979) that it is truly dimeric. Only one enzyme (abbreviated $\mathrm{Val}$ ) was found on gels which had been stained with valine-leucine as a substrate. This enzyme was distinguishable from the other peptidases by mobility differences and 
by the property that presumptive heterozygotes possessed three bands-suggesting that it is a functional dimer.

$L A P, L G G, G P D$ and $G-6-P D H$ were run on TC 100 buffer (Colgan, 1986). MPI was run on TEM 15 buffer and the other enzymes were run on TEM 50 buffer. All gels were run at $4^{\circ} \mathrm{C}$ with a potential drop of $200 \mathrm{~V}$ (over $17 \mathrm{~cm}$ ) between electrodes. Most systems were run for exactly 2 hours but $M P I$ and $G A-3-P D H$ were run for 2.5 hours and G-6-PDH and 6-PGDH for 3 hours.

The correspondence between allozymes on different gels was determined by running comparison gels with individuals displaying all of the variants found on a gel during the first series of runs. For $X$. pulex, LAP, LGG, Val, PGM and $G P I$ only were scored. Usually 219 individuals were typed for B. rostratus and 115 for $X$. pulex. Small differences from these numbers for some loci were due to a general weakness in staining of some mussels. In these only the strongest staining enzymes were scorable. The reduction in numbers for Val in B. rostratus was caused by the failure of two gels to stain at all, and the exhaustion of reserves of the relevant samples.

Generally, statistical analyses were performed using the SPSS program package. The "Crosstabs" subprogram was used to test for associations between loci. $\chi^{2}$ statistics were provided by this program for the raw data. However, the number of cells with low observed and expected numbers of observations made this a suspect procedure. So allozymes were pooled. Two subsequent analyses were performed. Firstly, allozymes were lumped into three classes. Secondly, the number of classes was reduced to two by further pooling. The pooling was done as follows. The three (or two) most common allozymes at a locus were identified. Then the rarer forms were incorporated into a class by associating them with the common allozyme which was nearest to them in mobility. Where $\chi^{2}$ analyses showed evidence of a significant association of genotypes between loci, a G-test (Sokal and Rohlf, 1969) was carried out to ensure that the significance was not due to a statistical artefact.

\section{RESULTS}

The data from the electrophoretic surveys are summarised in tables 1 and 2 . Table 1 presents details of the genotypic frequencies at the loci scored in Brachidontes rostratus and Xenostrobus pulex. It also includes a figure showing the observed frequency of the most common heterozygote as a percentage of the expected frequency of this type. The number of heterozygous classes, with an expected frequency of more than 3 per cent, which show a deficit from expected frequencies is also presented. Table 2 summarises the degree of association between pairs of loci in the two species. The $\chi^{2}$ probabilities of independence are given for the case where the allozymes were pooled into two classes. In the tables, allozymes are identified by numbers which are allocated in order of anodal mobility. For example, allozyme 1 has the highest anodal mobility of all representatives of an enzyme, migrating more rapidly than allozyme 2 which in turn migrates more rapidly than allozyme 3. The number of isoenzymes in heterozygotes was usually that which would be expected from the assumption that the enzyme has a particular functional form. That is, heterozygotes of monomeric enzymes possessed two bands, those of dimeric enzymes had three bands and those of tetramers had five bands. There was one exception to this pattern. One B. rostratus individual showed a twobanded heterozygous phenotype for Val. This could be due to heterozygosity of the normal form with a null allele (which does not prevent the dimer from functioning). If this is so, the estimated frequency of such nulls for this enzyme is less than 0.25 per cent. Nulls which prevent the dimer from functioning may still occur. No variants which could be ascribed to heterozygosity of a null and a normal allozyme were discovered for GPI-6$P G D H$ or $G P D$ which are known to be dimeric in other organisms (Harris and Hopkinson, 1977). There was no instance of an absence of staining for only one enzyme in an individual.

The data for GPD, GA-3-PDH and G-6-PDH are not included in table 2 because of the low level of variation found in these systems. Four allozymes, corresponding to those previously described (Colgan, 1981) were detected at the GPD locus. Allozyme 1 was found in a homozygote and in seven heterozygotes with the common allozyme 3 . Allozyme 2 was found in two heterozygotes with allozyme 3 and allozyme 4 in six heterozygotes with the common form. Only one (slow) variant was found (in heterozygous condition) for $G A-3$ $P D H$. Three allozymes were found at the G-6-PDH locus. The common type (2) is intermediate in mobility. Two 11 homozygotes, five 12 heterozygotes and four 23 heterozygotes were found.

A deficit of heterozygotes was seen for many genotypic classes: in B. rostratus 16 of the 24 types of heterozygotes with expected frequencies of more than 3 per cent showed such a deficit. Fewer heterozygotes than expected were observed for 9 
Table 1 Numbers of the various genotypes of enzyme loci in Brachidontes rostratus and Xenostrobus pulex. The actual numbers for a given genotype are written in the table. Enzyme abbreviations are explained in the text. The row labelled "Het \%" shows the observed frequency of the most common class of heterozygote as a percentage of its expected frequency. The row labelled "Def Classes" specifies the number of heterozygote classes (in the numerator), with an expected frequency of more than 3 per cent, which exhibit deficits. The denominator indicates the number of classes with expected frequencies greater than 3 per cent

\begin{tabular}{|c|c|c|c|c|c|c|c|c|c|c|c|c|}
\hline \multirow[b]{2}{*}{ Genotype } & \multicolumn{7}{|c|}{ B. rostratus } & \multicolumn{5}{|c|}{$X$. pulex } \\
\hline & $L A P$ & $L G G$ & Val & $P G M$ & $G P I$ & $M P I$ & 6-PGDH & $L A P$ & $L G G$ & Val & $P G M$ & $G P I$ \\
\hline 11 & 1 & 4 & & 3 & 2 & 4 & & & 2 & 3 & 2 & \\
\hline 12 & 1 & 12 & 4 & & & 18 & & & 10 & & & 5 \\
\hline 13 & 3 & 11 & 1 & 13 & 7 & 2 & 6 & & & 6 & 4 & 1 \\
\hline 14 & 3 & & 5 & 2 & & 18 & & 1 & 3 & & 1 & 8 \\
\hline 15 & 8 & & & 1 & & 10 & 2 & & & & & \\
\hline 22 & 7 & 104 & 78 & & 4 & 49 & 1 & 14 & 71 & 5 & 1 & 54 \\
\hline 23 & 11 & 50 & 6 & 7 & 35 & 8 & 10 & 9 & 3 & 11 & 15 & 1 \\
\hline 24 & 3 & 7 & 50 & 1 & & 42 & 2 & 4 & 7 & & & 22 \\
\hline 25 & 26 & & & 3 & & 16 & 1 & & & 2 & & 2 \\
\hline 26 & 4 & & & & & & 2 & & & & & 4 \\
\hline 33 & 6 & 26 & & 90 & 147 & 2 & 126 & 68 & 4 & 65 & 86 & \\
\hline 34 & 3 & 2 & 1 & 60 & 15 & 10 & 16 & 13 & 2 & 1 & 5 & 1 \\
\hline 35 & 27 & & & 10 & 6 & 2 & 45 & & 1 & 13 & & \\
\hline 36 & 4 & & & 3 & & & 3 & & & 1 & & 1 \\
\hline 44 & 13 & & 30 & 19 & 3 & 26 & & 4 & 7 & & 1 & 13 \\
\hline 45 & 14 & & 6 & 5 & & 6 & & & & & & 1 \\
\hline 46 & 3 & & & & & & & & & & & 1 \\
\hline 55 & 76 & & & 2 & & 6 & 5 & & & 4 & & \\
\hline 56 & 4 & & & & & & & & & 1 & & \\
\hline 57 & 1 & & & & & & & & & & & \\
\hline 66 & 1 & & & & & & & & & & & 1 \\
\hline Total & 219 & 216 & 191 & 219 & 219 & 219 & 219 & 113 & 110 & 112 & 115 & 115 \\
\hline Het $\%$ & 86 & 68 & 69 & 91 & 114 & 79 & 102 & 31 & 37 & 74 & 103 & 60 \\
\hline Def Classes & $5 / 6$ & $2 / 3$ & $1 / 1$ & $2 / 4$ & $0 / 2$ & $5 / 6$ & $1 / 2$ & $3 / 3$ & $2 / 2$ & $2 / 2$ & $1 / 2$ & $1 / 3$ \\
\hline
\end{tabular}

of 12 such classes in $X$. pulex. Deficiencies occurred in all seven of the enzymes shown in table 1although their degree seems more marked for the peptidases, both in terms of the amount of the deficiency in the most common heterozygote and of the number of classes exhibiting a deficit.

No significant association of functionallyrelated enzymes was detectable from the results shown in table 2. The only examples which approached statistical significance were $P G M$ with $G P I$ and MPI in B. rostratus. However, the null hypothesis of independence of genotypic frequencies at these loci was not rejected by any of the tests which were performed on the data. There were four possible associations in B. rostratus of pairs of loci which have no obvious functional relationship, and a further example of this was found in $X$. pulex. The four B. rostratus instances were (1) $L A P$ and $P G M$, where a G-test of the raw data (table 3 ) was significant at the 0.01 level; (2) $L G G$ and $G P I$, where the $\chi^{2}$ value in table 2 was significant but where a G-test on the pooling

Table 2 Probabilities of obtaining the observed two-locus genotypes by chance in pairwise tests of enzyme loci Brachidontes rostratus and Xenostrobus pulex. The probabilities are those calculated for the case when allozymes have been pooled into two types. The values above the diagonal refer to $B$. rostratus and those below this line to $X$. pulex

\begin{tabular}{llllllll}
\hline & LAP & LGG & Val & PGM & GPI & MPI & 6-PGDH \\
\hline LAP & & 0.39 & 0.41 & 0.68 & 0.80 & 0.28 & 0.87 \\
LGG & 0.90 & & 0.55 & 0.83 & 0.03 & 0.63 & 0.07 \\
Val & 0.80 & 0.63 & & 0.06 & 0.76 & 0.82 & 0.91 \\
$P G M$ & 0.24 & 0.81 & 0.52 & & 0.18 & 0.10 & 0.94 \\
$G P I$ & 0.27 & 0.53 & 0.03 & 0.68 & & 0.40 & 0.56 \\
$M P I$ & & & & & & & 0.98 \\
\hline
\end{tabular}


into three allelic classes was not so $(P>0 \cdot 25) ;(3)$ $L G G$ and 6-PGDH, were the G-test of the three allele pooling was significant at the 0.01 level; and (4) Val and PGM, where the G-test was again rejected at the 0.01 level. The example of significant interaction between loci in $X$. pulex was for GPI and Val. It is notable that no pair of enzymes was significantly associated in both species.

\section{DISCUSSION}

The determinants of the genotypic frequencies in marine mussels constitute one of the most puzzling problems in population genetics. In particular, the $L A P$ loci are invariably polymorphic and show disadvantage for all, or nearly all, classes of heterozygotes. Yet the polymorphisms do not appear to be transient. This situation can only be described as enigmatic. Since no convincing explanation of it has been developed, any new suggestion should be enthusiastically tested. The present work was prompted by the possibility, revealed by the theoretical work of Hastings (1982), that selective interactions with other loci might be responsible for the maintenance of polymorphisms with heterozygous disadvantage. A further stimulus was the report (Mitton and Koehn, 1973) of nonrandom association of the genotypic frequencies of a LAP and an AP locus in Mytilus edulis. Interactions between 10 loci (in four functional "groups") in Brachidontes rostratus and five loci (in two "groups") in Xenostrobus pulex were sought during the present study. Only seven of the loci in B. rostratus were sufficiently polymorphic to be useful in the analysis. Four of the twenty pairwise comparisons among these loci proved to be significant, as did one of the ten tests in $X$. pulex. None of the pairs exhibiting non-random associations comprised enzymes with clearly related functions. The significant associations which were found may derive from an epistatic constraint which has not been recognized. But it is more likely that they are due to linkage, to the Wahlund effect, or to some combination of these two factors. Non-random associations between $L A P$ and $P G M$ are known from M. edulis (Ahmad and Hedrick, 1985). Such an association was also found for B. rostratus. But it is not found in $X$. pulex.

The highly-dispersive nature of reproduction in mussels suggests that intra-population deviations from random mating would have small effects on allelic frequencies. And the Wahlund effect which would derive from inter-population variation of $L A P$ frequencies in $B$. rostratus is quite small (Colgan, 1981). But the size of this effect at other loci in B. rostratus and $X$. pulex has not been established. There are certainly no wholesale correlations between loci in either species in the present data. But it is known that differing estuarine and marine forms of $X$. pulex

Table 3 PGM and LAP genotypes in Brachidontes rostratus. The actual numbers of each two-locus genotype are written in the cells. The $L A P$ genotypes are represented by the rows of the table and the $P G M$ genotypes by the columns

\begin{tabular}{|c|c|c|c|c|c|c|c|c|c|c|c|c|c|c|}
\hline $\begin{array}{l}P G M \\
L A P\end{array}$ & 11 & 13 & 14 & 15 & 23 & 24 & 25 & 33 & 34 & 35 & 36 & 44 & 45 & 55 \\
\hline 11 & & & & & & & & 1 & & & & & & \\
\hline 12 & & & & 1 & & & & & & & & & & \\
\hline 13 & & & & & & & & & 1 & & 1 & 1 & & \\
\hline 14 & & & & & & & & 2 & 1 & & & & & \\
\hline 15 & & & & & & & & 5 & 1 & & & 2 & & \\
\hline 22 & & 1 & & & 1 & & & 4 & & 1 & & & & \\
\hline 23 & & & & & & 1 & & 7 & & 1 & & 1 & 1 & \\
\hline 24 & & & & & & & & & 2 & & & & 1 & \\
\hline 25 & & 3 & & & 4 & & & 6 & 8 & 1 & 1 & 3 & & \\
\hline 26 & & & & & & & & & 3 & & & 1 & & \\
\hline 33 & & & 1 & & 1 & & & 4 & & & & & & \\
\hline 34 & & & & & & & & 3 & & & & & & \\
\hline 35 & & 2 & 1 & & & & 1 & 12 & 7 & & & 3 & & 1 \\
\hline 36 & & 1 & & & 1 & & & 1 & 1 & & & & & \\
\hline 44 & & & & & & & & 6 & 4 & 2 & & & 1 & \\
\hline 45 & 1 & 2 & & & & & 1 & 3 & 7 & & & & & \\
\hline 46 & & 1 & & & & & & & 1 & & 1 & & & \\
\hline 55 & 2 & 3 & & & & & 1 & 34 & 21 & 5 & & 7 & 2 & 1 \\
\hline 56 & & & & & & & & 2 & 1 & & & 1 & & \\
\hline 57 & & & & & & & & & 1 & & & & & \\
\hline 66 & & & & & & & & & 1 & & & & & \\
\hline
\end{tabular}


do exist (May and McPherson, 1958). The amount of interbreeding between the forms has not been investigated. So, there is potential for a large Wahlund effect in this species.

The results reported in this paper suggest that epistatic interactions between loci are not responsible for the maintenance of the aminopeptidase polymorphisms of marine mussels. A difficulty with unconditional acceptance of this suggestion is that the locus or loci which are important in the interaction with those which have been typed might not have been identified. For instance, variation in the active peptidase which was not scorable in $B$. rostratus may be highly correlated with variation in one of the scored loci-although, if it were correlated with more than one, then a secondary association between them would be expected. There may be other peptidases or proteases which are also involved in the determination of fitnesses at the scored loci. Whilst this suggestion remains a possibility, it should be noted that all of the peptidase loci in the present study generally show deficits of heterozygotes. These loci might all interact with one other (untyped) locus or they might severally interact with one or more distinct unidentified loci. In either case, however, it would have to be argued that interactions between the three identified loci in each species are not typical of all those between peptidases, as no significant associations between the loci were detected.

There may yet be discovered to be circumstances where the frequencies of a given $L A P$ or $A P$ polymorphism could be explained by interaction with another locus. But the wide range of genotypic frequencies in the diversity of studied species requires that the explanation of the maintenance of loci with a deficit of heterozygotes in marine mussels be quite general. The results reported here cast some doubt on the generality of selective interactions between loci as the cause of the maintenance of polymorphisms in mussels. A number of other possible, though mostly implausible, explanations of the deficits were mentioned in the Introduction. Their true cause may be something as trivial as the repression of an allele in genetic heterozygotes. But it may also be that their underlying basis is a totally novel phenomenon. The elucidation of such a phenomenon would have major importance for the whole of population genetics.

Acknowledgements I wish to thank Dr G. Brand of the Marine Science Laboratories, Victorian Department of Conservation, Queenscliff for his generous effort in collection of the mussels and $\mathrm{Mr} \mathrm{D}$. Rowell for explanation of the operation of the local computer system.

\section{REFERENCES}

ADAMKEWICZ, L., TAUB, S. R. AND WALL, J. R. 1984. Genetics of the clam Mercenaria mercenaria. I. Mendelian inheritance of allozyme variation. Biochem. Genet., 22, 215-219.

AHMAD, M. AND HEDRICK, P. W. 1985. Electrophoretic variation in the common mussel, Mytilus edulis: random association of alleles at different loci. Heredity, 55, 47-51.

BEAUMONT. A. R., BEVERIDGE, C. M. AND BUDD, M. D. 1983. Selection and heterozygosity within single families of the mussel Mytilus edulis (L.). Marine Biol. Lett., 4, 151-161.

COLGAN, D. J. 1980. Theoretical and Practical Studies on Genetic Fitness. Ph.D. Thesis, University of Melbourne, Melbourne.

COLGAN, D. J. 1981. Spatial and temporal variation in the genotypic frequencies of the mussel Brachidontes rostratus. Heredity, 46, 197-208.

COLGAN, D. J. 1984. The effects of linked loci on estimates of relative viability derived from pedigree analysis. Heredity, $53,69-77$.

COLGAN, D. J. 1986. Developmental changes in the isoenzymes controlling glycolysis in the acridine grasshopper, Caledia captiva. W. Roux Arch. Dev. Biol. 195, 197 201.

FOLTZ, D. W. 1986. Null alleles as a possible cause of heterozygote deficiencies in the oyster Crassostrea virginica and other bivalves. Evolution, 40, 869-870.

GARTHWAITE, R. 1986. The Genetics of Californis populations of Guekensia demissa (Dillwyn) (Mollusca): further evidence on the selective importance of leucine aminopeptidase variation in salinity acclimation. Biol. J. Linn, Soc., 28, 343-358.

GARTNER-KEPKAY, K. E., DICKIE, L. M., FREEMAN, K. R. AND ZOLROS, E. 1980. Genetic differences and environments of mussel populations in the Maritime provinces. Can. J. Fish. Aquat. Sci., 37, 775-782.

HARRIS, H. AND HOPKINSON, D. A. 1977. Handbook of Enzyme Electrophoresis in Human Genetics. North-Holland, Amsterdam.

HASTINGS, A. 1982. Unexpected behavior in two-locus genetic systems: an analysis of marginal underdominance at a stable equilibrium. Genetics, 102, 129-138.

HilbisH, T. J. 1985. Demographic and temporal structure of an allele frequency cline in the mussel Mytilus edulis. Mar. Biol., 86, 163-171.

KARTAVTSEV, Y. F. 1978. Genetic variability of a bivalve mollusk, the mussel Crenomytilus grayanus. Soviet Genetics, $14,186-190$.

KoEHN, R. K., Milkman, R. AND mitton, J. B. 1976. Population genetics of marine pelecypods. IV. Selection, migration and genetic differentiation in the blue mussel Mytilus edulis. Evolution, 30, 2-32.

LEVINTON, J. S. AND SUCHANEK, T. H. 1978. Geographic variation, niche breadth and genetic differentiation at different geographic scales in the mussels Mytilus edulis and Mytilus californianus. Mar. Biol., 49, 369-376.

LEWONTIN, R. C. 1958. A general method for investigating the equilibrium of gene frequency in a population. Genetics, $43,419-434$.

MALLET, A. L. ZOUROS, E., GARTNER-KEPKAY, K. E., FREEMAN, K. R. ANI DICKIE, L. M. 1985. Larval viability and heterozygote deficiency in populations of marine bivalves: evidence from pair matings of mussels. Mar. Biol., 87, $165-172$.

MAY, W. L. AND MACPHERSON, J. H. 1958. An Illustrated Index of Tasmanian Shells, D. E. Wilkinson, Government Printer, Tasmania. 
MITTON, J. B. AND KOEHN, R. K. 1973. Population genetics of marine pelecypods. III. Epistasis between functionally related isoenzymes of Mytilus edulis. Genetics, 73, 487-496.

SKIBINSKI, D. O. F., BEARDMORE, J. A. AND CROSS, T. F. 1983. Aspects of the population genetics of Mytilus (Mytilidae; mollusca) in the British Isles, Biol. J. Linn. Soc., 19, 137-183. SOKAL, R. R. AND ROHLF, F. J. 1969. Biometry, W. H. Freeman, San Francisco.

THEISEN, B. F. 1978. Allozyme clines and evidence for strong selection in three loci in Mytilus edulis L. (Bivalvia) from Danish waters. Ophelia, 17, 135-142.
YOUNG, J. P. W., KOEHN, R. K. AND ARNHEIM, N. 1979. Biochemical characterization of "Lap", a polymorphic aminopeptidase from the blue mussel Mytilus edulis. Bioch. Genet., 17, 305-323.

ZOUROS, E. AND FOLTZ, D. W. 1984. Possible explanations of heterozygote deficiency in bivalve molluscs. Malacologia, $25,583-591$. 